\title{
Deep Venous Thrombosis in Pregnancy
}

\author{
Aarti Mishra ${ }^{1}$, Saunitra Inamdar ${ }^{2}$
}

\begin{abstract}
Deep venous thrombosis (DVT) is one of the rare yet important causes of maternal morbidity and mortality. In all, 2-3\% of cases are fatal. Since pregnancy is a hypercoagulable state, it is more prone to develop DVT than normal females. Patient presents with swelling, pain, and warmth in the affected limb. Diagnosis is made by Doppler. Pulmonary embolism is the most fatal complication. First line of treatment is by broadspectrum antibiotic and anticoagulants. Here, we review two cases: a 23-year-old primi with 32.3 weeks GA with acute history of DVT and a 30-year-old G4P1L1A2 with chronic DVT in right leg and acute DVT in left leg. We will be focusing on the clinical presentations, investigations, management of the case, the current state of literature, and the current standard treatment.
\end{abstract}

Keywords: Deep venous thrombosis in pregnancy, Procoagulant state, Pulmonary embolism, Venous thrombosis.

Journal of South Asian Federation of Obstetrics and Gynaecology (2020): 10.5005/jp-journals-10006-1759

\section{INTRODUCTION}

Deep venous thrombosis (DVT) is one of the many causes of maternal mortality. ${ }^{1}$ Its incidence is about 1/1,000 deliveries of which $2-3 \%$ are fatal. Normally, it is not as fatal, but during pregnancy, the risk increases 5-10 times, and in cesarean section, the risk further increases by 5 - to 10 -fold. It is highest during labor and in the immediate postpartum period, where it is five times greater than that during pregnancy.

DVT in pregnancy, although a rare disease, is more common in pregnant state than nonpregnant.

Pregnancy has all three components of Virchow's triad: venous stasis, endothelial damage, and hypercoagulability. ${ }^{1}$

Venous stasis results from a decrease in venous tone due to hormones and the obstruction of venous flow by enlarging uterus. ${ }^{1}$ A reduction in venous flow of approximately $50 \%$ occurs in the legs by $25-29$ weeks of gestation and lasts until approximately 6 weeks postpartum, after which time normal venous velocities return.

Certain conditions have higher risk of DVT in pregnancy, such as inherited or acquired thrombophilias, a previous history of thrombosis, antiphospholipid syndrome, lupus, heart disease, and sickle cell disease. When these are present, there is need for prophylactic anticoagulation.

Pulmonary embolism is the most feared complication of DVT.

The standard care for this condition is anticoagulant therapy.

\section{Case Description}

\section{Case 1}

A 23-year-old primigravida with 32.3 weeks GA with DVT in left leg with mild anemia was admitted in Acharya Vinoba Bhave Rural Hospital 1.5 months earlier. She came with swelling and pain in left leg since 8 days.

\section{General Examination}

Patient is well nourished, averagely built, afebrile, $\mathrm{P}-80 \mathrm{bpm}$, blood pressure (BP) 110/70 mm Hg, pallor absent, and edema present.

Patient was brought to the casualty on a wheelchair, as she was unable to walk due to the swelling in her legs (left $>$ right).

\section{Systemic Examination}

CNS: Patient was conscious, alert, and well oriented to time, place, and person.
${ }^{1}$ Department of Obstetrics and Gynaecology, Datta Meghe Institute of Medical Sciences (Deemed to be University), Maharashtra, India

${ }^{2}$ Department of Obstetrics and Gynecology, Acharya Vinoba Bhave Rural Hospital, Wardha, Maharashtra, India

Corresponding Author: Aarti Mishra, Department of Obstetrics and Gynaecology, Datta Meghe Institute of Medical Sciences (Deemed to be University), Maharashtra, India, Phone: +91 8979012672, e-mail: jiamshr77@gmail.com

How to cite this article: Mishra A, Inamdar S. Deep Venous Thrombosis in Pregnancy. J South Asian Feder Obst Gynae 2020;12(1):48-50.

Source of support: Nil

Conflict of interest: None

Respiratory: $\mathrm{B} / \mathrm{L}$ air entry present, $\mathrm{B} / \mathrm{L}$ chest clear.

CVS: s1 and s2 heard, no added sounds or murmur heard

P/A: Liver/spleen not palpable

Uterus: 34-week size, relaxed, cephalic, FHS+/r/145 bpm

$\mathrm{P} / \mathrm{v}$ : No indication of $\mathrm{p} / \mathrm{v}$ thus not performed

L/E:

Left-side foot and leg upto the knee red, swollen, shiny

Dilated tortuous vein present

On palpation: warm on touch, tender

\section{Investigations}

Hb 9 gm\%, TLC 10, 600 cells/cumm, PLT 2.41 lakh/cumm, Na 144, $\mathrm{K} 4.4$, urea 17, creatinine 0.5 , ALP 22, ALT 9, AST 28, total protein 6.4, albumin 3.2, total bilirubin 0.6 , conjugated bilirubin 0.3 , globulin 3.2, uric acid 3.8, LDH 227, PT(INR) 01.02 (12.7), APTT 30.5, FBS 76, anti-phospholipid antibody 1.47, anti-dsDNA 0.34, ANA 1.75 AU/mL.

Her left leg Doppler revealed near-total echogenic thrombosis involving the lower limb from the common iliac vein up to the popliteal vein with involvement of great vein of saphenous with loss of compressibility. The popliteal blood flow is normal (Fig. 1).

Her ANC scan shows SLIUF of 35.5 weeks GA and EFW OF $2.8 \mathrm{~kg}$ with maternal right-sided hydronephrosis.

Doppler: Right uterine artery shows early diastolic notch with high resistance flow; rest of the vessels were normal.

She was given strict bedrest and was in HDU; PT INR was monitored every week.

(0) The Author(s). 2020 Open Access This article is distributed under the terms of the Creative Commons Attribution 4.0 International License (https://creativecommons. org/licenses/by-nc/4.0/), which permits unrestricted use, distribution, and non-commercial reproduction in any medium, provided you give appropriate credit to the original author(s) and the source, provide a link to the Creative Commons license, and indicate if changes were made. The Creative Commons Public Domain Dedication waiver (http://creativecommons.org/publicdomain/zero/1.0/) applies to the data made available in this article, unless otherwise stated. 


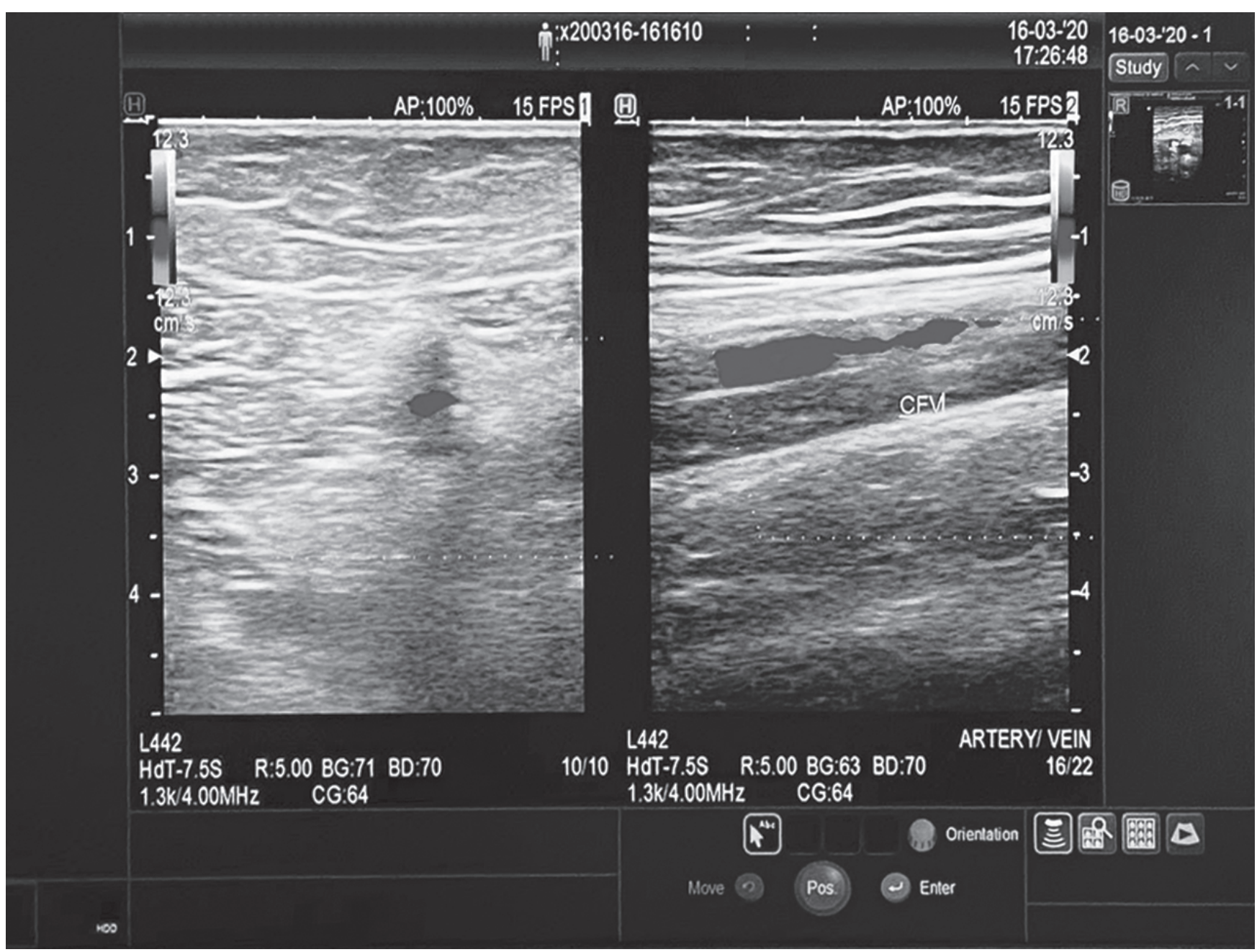

Fig. 1: Doppler of lower limb showing blood file changes suggestive of DVT

She was started on: inj augmentin $1.2 \mathrm{~g}$ intravenously (IV) 12 hourly, inj pan 40 IV 12 hourly, inj Lomoh $0.6 \mathrm{mh}$ s/c once daily, tab chymoral forte twice daily, $\mathrm{MgSO}_{4}$ dressing on the DVT site, and inj dexa $6 \mathrm{mg} 6$ hourly 4 doses were given. She was also transfused on unit whole blood.

Doppler done postprocedure revealed hypoehoic luminal content in veins of left lower limb extending from common iliac vein up to the popliteal vein with partial recanalization.

After Doppler, she was shifted to inj Lomoh $0.6 \mathrm{mg} \mathrm{s} / \mathrm{c}$, tab duvadilan $10 \mathrm{mg}$ TDS, tab Udiliv $300 \mathrm{mg}$ twice daily, tab polaramine $2 \mathrm{mg}$ once daily sos, tab AFI plus twice daily, tab vasolarge twice daily, tab chymoral forte twice daily, tab iron twice daily, tab calcium twice daily, L/A calosoft lotion twice daily, tab limcee twice daily, and tab zincovit twice daily.

At 37 weeks, she was taken up for a planned cesarean section and delivered a female child $/ 2.4 \mathrm{~kg} / \mathrm{mother}$ 's side. She was discharged on tab Acitrom $4 \mathrm{mg}$ and also adviced follow-up after 7 days.

\section{Case 2}

A 30-year-old patient G4P1L1A2 with 7.6 weeks GA was admitted with DVT in the right leg with moderate anemia to AVBRH on March 28,2020 . She came with complaints of pain and edema in right leg since 3 days.
She had a history of DVT in left leg after 40 days of her first delivery for which IVC filter inserted in 2012 (IVC filter in situ); since then, the patient was on tab warfarin $5 \mathrm{mg}$ and $7.5 \mathrm{mg}$ alternatively for the past 9 years.

Patient stopped medication in October after IR consultation and was started on tab Homocheck for 2 months.

\section{General examination}

Patient is well nourished, averagely built, afebrile, P-80 bpm, BP $110 / 70 \mathrm{~mm} \mathrm{Hg}$, pallor absent, and edema present.

\section{Systemic Examination}

CNS: Patient was conscious, alert, and well oriented to time, place, and person.

Respiratory: $B / L$ air entry present, $B / L$ chest clear.

CVS: $\mathrm{s} 1, \mathrm{~s} 2$ heard, no added sounds or murmur heard

P/A: liver spleen not palpable

soft

$\mathrm{P} / \mathrm{v}$ : No indication of $\mathrm{p} / \mathrm{v}$ thus not performed

L/E:

Right-side foot was red, swollen, and shiny.

Dilated tortuous vein was present.

On palpation: warm on touch and tender 


\section{Investigations}

Hb 8.3 gm\%, TLC 6000 cells/cumm, PLT 1.11 lakh/cumm, Na 140, K 3.8, urea 15, creatinine 0.7, ALP 89, ALT 13, AST 20, total protein 7.1, albumin 3.8, total bilirubin 0.4 , conjugated bilirubin 0.1 , globulin 3.3, uric acid 3.6, LDH 205, PT(INR) 01.07 (13.4), APTT 35.4 RBS 76.

Her right leg Doppler revealed partial echogenic thrombosis involving the lower limb from the common iliac vein up to the popliteal vein. The popliteal blood flow is normal.

Her ANC scan shows SLIUF of CRL 22MM corresponding to 8.6 weeks GA. She was given strict bedrest and was in HDU. She was also transfused on unit whole blood.

She was started on inj Lomoh $0.6 \mathrm{~g} \mathrm{S/C} \mathrm{BD,} \mathrm{tab} \mathrm{Acitrom} 1 \mathrm{mg}$ OD, T Azee 500 mg OD, T CTax 200 mg BD, Tab Pan 40, Tab Metro $400 \mathrm{mg}$ ER.

Medication termination of pregnancy was done on March 30, 2020.

She was discharged after 5 days on tab Azee $500 \mathrm{mg}$ OD X 2 days, Tab Acitrom $3 \mathrm{mg}$ OD at 4:00 pm.

She was adviced follow-up after 1 week and adviced repeat test of PT INR and APTT after 1 week.

\section{Discussion}

Deep venous thrombosis is one of the rare causes of maternal deaths. It is a procoagulant state. There is alteration between prothrombotic and anticoagulant factors resulting in increase in fibrin deposition and decreases fibrinolysis. At around 25-29 weeks, the flow venous in the lower limb is reduced by $50 \%$.

Important risk factors are multiparity, puerperium, postoperative periods, infections, neoplasia, systemic lupus erythromatous, and hypercoagulability states. ${ }^{2}$

Diagnosis can be made by various methods such as compression USG or Doppler that has a sensitivity of $97-100 \%$ and a specificity of $98-99 \%$, while CECT and magnetic resonance imaging can confirm the diagnosis and reveal the extent of thrombosis. ${ }^{1}$

Pulmonary embolism (PE) is the most fatal complication occurring in $13 \%$ of cases.

Broad-spectrum antibiotics and IV heparin or low-molecularweight heparin $(\mathrm{LMWH})$ is usually the first line of management. Anticoagulants once started must be continued for 3-6 months.

Even after proper treatment, embolism may develop. ${ }^{1}$

In case of extensive DVT, caval filters are recommended.

Switching from LMWH to unfractionated heparin is done during the end of pregnancy or sooner if delivery is early. ${ }^{1}$
Anticoagulants should be restarted within 12 hours after normal delivery and 24 hours after cesarean delivery to reduce the bleeding complications.

The use of pneumatic compression bands are advised during labor or before cesarean delivery in women, where anticoagulant therapy has been put on hold for some time and should be left in place until the patient is ether mobile again or/and until anticoagulation is restarted. ${ }^{3}$ Warfarin is the drug of choice during breastfeeding. Warfarin is also recommended postdelivery for 3-4 months in women developing DVT in the current pregnancy. ${ }^{1}$

Estrogen-containing OCPs are contraindicated in women with history of thrombophilia or thrombosis. Contraceptive pill of choice for such patients are progesterone-only pills. ${ }^{3,4}$

\section{Conclusion}

Pregnancy is a hypercoagulable state, and it has all the components of Virchow's triad. ${ }^{2}$

Risk factors are history of thrombosis, thrombophilia, etc. Not all women require anticoagulant therapy, only the ones with history or current complaint of thrombosis or with thrombophilia and a history poor pregnancy outcome require the same. Anticoagulation in pregnancy have both maternal and fetal side effects. Thus, considering fetal side effects, the preferred anticoagulant in pregnancy are heparin compounds. ${ }^{3}$

And during breastfeeding, the preferred anticoagulant is warfarin.

At the time of delivery, anticoagulation should be shifted from LMWH to unfractionated hepatin to reduce the risk of bleeding complications along with minimizing the risk of thrombosis.

\section{References}

1. Srivastava M, Bhatnagar P, Gupta M. Deep vein thrombosis in postpartum case of caesarean section: a case report. Int J Sci Stu 2015;3(1).

2. James $A H$. Venous thromboembolism in pregnancy. Arterioscler Thromb Vasc Biol 2009;29(3):326-331. DOI: 10.1161/ ATVBAHA.109.184127.

3. RiskFactorsforandClinical Management ofVenous Thromboembolism During Pregnancy - Hematology \& Oncology [Internet]. [cited 2020 Apr 26]. Available from: https://www.hematologyandoncology.net, https://www.hematologyandoncology.net/archives/july-2019/riskfactors-for-and-clinical-management-of-venous-thromboembolismduring-pregnancy/.

4. Devis $P$, Knuttinen MG. Deep venous thrombosis in pregnancy: incidence, pathogenesis and endovascular management. Cardiovasc Diagn Ther 2017;7(S3):S309-S319. DOI: 10.21037/cdt.2017. 10.08 . 